DFTT $15 / 2008$

EPHOU 08-003

May, 2008

\title{
Matrix formulation of superspace on 1D lattice with two supercharges
}

\author{
Sergio Arianos ${ }^{\mathrm{a} *}$, Alessandro D’Adda ${ }^{\mathrm{a}}$, Alessandra Feo ${ }^{\mathrm{a}}$, Noboru Kawamoto ${ }^{\mathrm{b}}$ \\ and Jun Saito ${ }^{\mathrm{b}}$ \\ a INFN sezione di Torino, and \\ Dipartimento di Fisica Teorica, Universita di Torino \\ I-10125 Torino, Italy \\ b Department of Physics, Hokkaido University \\ Sapporo, 060-0810 Japan
}

\begin{abstract}
Following the approach developed by some of the authors in recent papers and using a matrix representation for the superfields, we formulate an exact supersymmetric theory with two supercharges on a one dimensional lattice. In the superfield formalism supersymmetry transformations are uniquely defined and do not suffer of the ambiguities recently pointed out by some authors. The action can be written in a unique way and it is invariant under all supercharges. A modified Leibniz rule applies when supercharges act on a superfield product and the corresponding Ward identities take a modified form but hold exactly at least at the tree level, while their validity in presence of radiative corrections is still an open problem and is not considered here.
\end{abstract}

\section{Introduction}

A consistent formulation of supersymmetry on the lattice is a long standing problem (see [1] and references therein). Older papers on the subject make use of schemes that break supersymmetry explicitly on the lattice and recover it in the continuum limit. This however leads in general to fine tuning problems. In recent years a number of approaches that allow to preserve exactly one

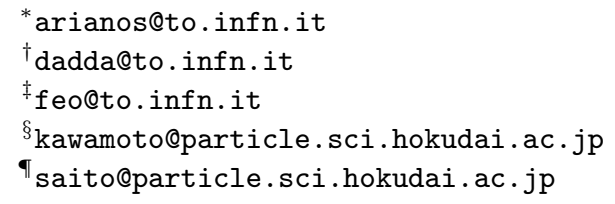


supersymmetry on the lattice in theories with an extended supersymmetry have been proposed $[2,3,4]$. A more ambitious approach, which we follow in the present paper, aiming to preserve exactly all supersymmetries in some extended supersymmetric model, was also proposed [5, $6,7,8]^{1}$. See also related works on the subject $[11,12,13,14,15,16]$ and reviews at lattice conferences $[17,18,19,1,20]$.

There are two important well known obstacles in formulating a supersymmetric lattice theory: Firstly, Poincaré invariance is lost on the lattice, thus supersymmetry is lost as well. In particular derivatives are replaced on the lattice by finite differences which do not satisfy the Leibniz rule when applied to a product of fields [11]. Secondly, a naive regularization leads to the doubling problem. This results in the wrong number of degrees of freedom and therefore in a violation of the balance between fermions and bosons.

The approach we are going to follow makes use of Dirac-Kähler twisting procedure developed in the continuum formulation [21]. Its main feature however is the use of an extended lattice where the standard links, corresponding to the discrete elementary translations on the lattice, are implemented by "fermionic" links that correspond to the action of supersymmetry charges. The need for these extra links is the result of the modified "shifted" Leibniz rules, that both translations and supersymmetry transformation have for consistency to satisfy on a lattice when acting on a product of (super)fields. The structure of the extended lattice thus reflects the structure of the twisted supersymmetry algebra, and a solution is found only for some specific extended superalgebras which are consistent with the twisted supersymmetry algebra, like the $N=2$ superalgebra for $D=2[5,7]$, the $N=4$ superalgebra for $D=3[8,22]$, and the $N=4$ superalgebra for $D=4[7]$. The above mentioned two obstacles are overcome in this formulation: The twisted lattice supersymmetry algebra with the difference operator is realized algebraically by introducing the shifted Leibniz rule. The extra degrees of freedom of species doublers of the lattice chiral fermions, which were usually identified as flavor degrees of freedom, can be identified as extended supersymmetry degrees of freedom. From the consistency, the supersymmetry charges are in this approach associated to links, rather than to sites. In connection with this point a number of criticisms were put forward [23, 24], with the claim that the link nature of supercharges leads to inconsistencies and ambiguities in the definition of the supersymmetry transformations. Very recently [26] it was shown that this approach fits within the scheme of Kaplan's orbifold formulation [2] and the actual invariance of the action proposed in [7] under all susy charges has been questioned.

In order to investigate the above issues we considered a simple one dimensional supersymmetric model with two supercharges [25], the same model already considered by Bruckmann and de Kok in [23]. In this context we develop a matrix representation of superfields on the lattice, already anticipated in [27], and show using the superfield formalism that the lattice action is invariant under all supersymmetry charges and that supersymmetry transformations are consistently and unambiguously determined in terms of superfield on the lattice, thus overcoming the objections of [23]. Since (super)symmetry transformations on the lattice make use of modified Leibniz rules, supersymmetry cannot be expressed simply as a change of variables in the functional integral and the existence of Ward identities showing that it is exactly preserved at the quantum level is not obvious. We then decided to investigate tree level Ward identities for the free action (including the mass term) and found that exact Ward identities hold in that case showing that lattice supersymmetry is really a symmetry of the action. Such Ward identities are "modified" in a way that is reminiscent of the modified Leibniz rules, and reflect the particular

\footnotetext{
${ }^{1}$ while for the $N=1$ Wess-Zumino model in 4 dimensions an exact lattice formulation has been achieved using the Ginsparg-Wilson formulation [9] which gives exact Ward-Takahashi identities at fixed lattice spacing [10].
} 
nature of the lattice symmetry. The question of whether modified Ward identities can be derived also in the case of interaction, namely at loop level, is still unanswered and is being investigated.

The paper is organized as follows: In Section 2 we describe the one dimensional $N=2$ supersymmetric model in the continuum. In Section 3 we give some general properties of one dimensional lattice theories and in particular the modified Leibniz rule obeyed by finite difference operator when acting on a product of fields on the lattice. The generalization of this rule to the supersymmetric case is one of the main ingredients of our formulation. The superfield formulation of the $N=2$ one dimensional supersymmetric model on the lattice, including the algebra and the supersymmetry transformations, is presented in Section 4 . In Section 5 we construct the supersymmetric lattice action in terms of superfields and also in terms of the component fields. Section 6 is dedicated to the study of the modified Ward identities and some examples to tree level are given. Conclusions on Section 7.

\section{The model}

The model we are going to discuss in this paper is a one dimensional supersymmetric model with two supersymmetry charges [25], whose lattice formulation was recently discussed in [23]. Its supersymmetry algebra is given, in terms of Majorana SUSY charges $Q_{1}$ and $Q_{2}$ by:

$$
\begin{aligned}
Q_{1}^{2} & =Q_{2}^{2}=P_{x}, \\
\left\{Q_{1}, Q_{2}\right\} & =0, \quad\left[P_{x}, Q_{1}\right]=\left[P_{x}, Q_{2}\right]=0,
\end{aligned}
$$

where $P_{x}$ is the generator of translations in the one-dimensional space-time coordinate $x^{2}$ :

$$
P_{x}=\frac{\partial}{\partial x}
$$

A superspace representation of the algebra may be given in terms of two Grassmann odd, real coordinates $\theta_{1}$ and $\theta_{2}$, namely:

$$
Q_{1}=\frac{\partial}{\partial \theta_{1}}+\theta_{1} \frac{\partial}{\partial x}, \quad Q_{2}=\frac{\partial}{\partial \theta_{2}}+\theta_{2} \frac{\partial}{\partial x} .
$$

The field content of the theory is described by a hermitian superfield $\Phi\left(x, \theta_{1}, \theta_{2}\right)$ :

$$
\Phi\left(x, \theta_{1}, \theta_{2}\right)=\varphi(x)+i \theta_{1} \psi_{1}(x)+i \theta_{2} \psi_{2}(x)+i \theta_{2} \theta_{1} D(x)
$$

where $\psi_{1}$ and $\psi_{2}$ are Majorana fermions. The supersymmetry transformations of the superfield $\Phi$ are given by:

$$
\delta_{j} \Phi=\left[\eta_{j} Q_{j}, \Phi\right] \quad j=1,2,
$$

where $\eta_{i}$ are the Grassmann odd parameters of the transformation. In terms of the component fields eq. (2.5) reads:

$$
\begin{aligned}
\delta_{j} \varphi & =i \eta_{j} \psi_{j}, \\
\delta_{j} \psi_{k} & =i \delta_{j, k} \eta_{j} \partial_{x} \varphi+\epsilon_{j k} \eta_{j} D, \\
\delta_{j} D & =-\epsilon_{j k} \eta_{j} \partial_{x} \psi_{k} .
\end{aligned}
$$

\footnotetext{
${ }^{2}$ The coordinate $x$ is the Wick rotated time coordinate $t \rightarrow i x$, so that we are actually describing a euclidean formulation.
} 
It is important to note that since the supersymmetry transformations are defined in (2.5) as commutators, supersymmetry transformations of superfields products obey ordinary Leibniz rule:

$$
\delta_{i}\left(\Phi_{1} \Phi_{2}\right)=\left(\delta_{i} \Phi_{1}\right) \Phi_{2}+\Phi_{1}\left(\delta_{i} \Phi_{2}\right) .
$$

In order to write a supersymmetric action we need to introduce the superderivatives, defined as

$$
D_{j}=\frac{\partial}{\partial \theta_{j}}-\theta_{j} \frac{\partial}{\partial x}
$$

which anticommute with the supersymmetry charges $Q_{j}$ and satisfy the algebra:

$$
D_{j}^{2}=-\frac{\partial}{\partial x}, \quad\left\{D_{1}, D_{2}\right\}=0 .
$$

The supersymmetric action can then be defined in terms of the superfield $\Phi$ as:

$$
\int d x d \theta_{1} d \theta_{2}\left[\frac{1}{2} D_{2} \Phi D_{1} \Phi+i V(\Phi)\right]
$$

where $V(\Phi)$ is a superpotential that we will choose, as in [23], to be of the form:

$$
V(\Phi)=\frac{1}{2} m \Phi^{2}+\frac{1}{4} g \Phi^{4} .
$$

By integrating over $\theta_{1}$ and $\theta_{2}$ in (2.12) one can obtain the action written in terms of the component fields, namely:

$$
\begin{gathered}
S=\int d x\left\{\frac{1}{2}\left[\left(\partial_{x} \varphi\right)^{2}-D^{2}-\psi_{1} \partial_{x} \psi_{1}-\psi_{2} \partial_{x} \psi_{2}\right]\right. \\
\left.-m\left(i \psi_{1} \psi_{2}+D \varphi\right)-g\left(3 i \varphi^{2} \psi_{1} \psi_{2}+D \varphi^{3}\right)\right\} .
\end{gathered}
$$

The auxiliary field $D$ appears quadratically in (2.14) and can therefore be integrated out, leading to the on-shell action:

$$
\begin{aligned}
S & =\int d x\left\{\frac{1}{2}\left[\left(\partial_{x} \varphi\right)^{2}-\psi_{1} \partial_{x} \psi_{1}-\psi_{2} \partial_{x} \psi_{2}+m \varphi^{2}-2 i m \psi_{1} \psi_{2}\right]\right. \\
& \left.+m g \varphi^{4}+\frac{g^{2}}{2} \varphi^{6}-3 i g \varphi^{2} \psi_{1} \psi_{2}\right\} .
\end{aligned}
$$

\section{One dimensional lattice and modified Leibniz rules}

We discuss in this section some general properties of one dimensional lattice theories, without introducing supersymmetry, and in particular the modified Leibniz rule obeyed by the finite difference operator when acting on a product of fields on the lattice. The generalization of this rule to the supersymmetric case is one of the main ingredients of our formulation. To begin with, let us introduce a matrix notation for fields on a one dimensional lattice.

Consider a one dimensional lattice with $N$ sites and periodic boundary conditions. Let $a$ be the lattice spacing. The $N$ sites of the lattice will be labeled by a coordinate $\boldsymbol{x}=r a$ where $r$ is an integer modulo $N$. The lattice is compactified, in the sense that the points $\boldsymbol{x}$ and $\boldsymbol{x}+L$ are identified, $L$ being the lattice size:

$$
L=a N
$$


A scalar field $\varphi$ on the lattice is defined by a set of $N$ numbers $\varphi_{r}(r=1,2, \cdots, N)$ which give the value on the field on the lattice sites of coordinate $\boldsymbol{x}=\mathrm{ra}$ :

$$
\varphi(\boldsymbol{x})=\varphi(r a) \equiv \varphi_{r}
$$

The $N$ numbers $\varphi_{r}$ can be regarded as the eigenvalues of a $N \times N$ diagonal matrix $\varphi$ :

$$
\boldsymbol{\varphi}=\left(\begin{array}{cccccc}
\varphi_{1} & 0 & 0 & 0 & \cdots & 0 \\
0 & \varphi_{2} & 0 & 0 & \cdots & 0 \\
0 & 0 & \varphi_{3} & 0 & \cdots & 0 \\
\vdots & \vdots & \vdots & \vdots & \ddots & \vdots \\
0 & 0 & 0 & 0 & \cdots & \varphi_{N}
\end{array}\right)
$$

whose rows and columns are in one to one correspondence with the sites of the lattice. Notice that the ordering of the rows and columns is the same as the one of the lattice sites, so that neighboring eigenvalues correspond to the values of the field in neighboring sites. Derivatives are replaced on the lattice by finite differences:

$$
\frac{\partial \varphi(x)}{\partial x} \rightarrow \frac{1}{a} \partial_{+} \varphi(\boldsymbol{x}) \equiv \frac{1}{a}(\varphi(\boldsymbol{x})-\varphi(\boldsymbol{x}-a))
$$

or, with the notations of eq. (3.2):

$$
\left(\partial_{+} \varphi\right)_{r}=\left(\varphi_{r}-\varphi_{r-1}\right)
$$

The breaking of the translational invariance due to the discrete nature of the lattice results into a violation of the Leibniz rule when the finite difference of a product of two functions is considered. As discussed in detail in ref. [5], to which we refer for a more exhaustive treatment, a modified Leibniz rule holds in place of the usual one:

$$
\left(\partial_{+} \varphi \psi\right)_{r}=\left(\partial_{+} \varphi\right)_{r} \psi_{r}+\varphi_{r-1}\left(\partial_{+} \psi\right)_{r}
$$

In matrix notation finite differences may be represented using the shift matrices $\boldsymbol{\Delta}_{+}$and $\boldsymbol{\Delta}_{-}$:

$$
\boldsymbol{\Delta}_{+}=\left(\begin{array}{cccccc}
0 & 1 & 0 & 0 & \cdots & 0 \\
0 & 0 & 1 & 0 & \cdots & 0 \\
0 & 0 & 0 & 1 & \cdots & 0 \\
\vdots & \vdots & \vdots & \vdots & \ddots & \vdots \\
0 & 0 & 0 & 0 & \cdots & 1 \\
1 & 0 & 0 & 0 & \cdots & 0
\end{array}\right), \quad \boldsymbol{\Delta}_{-}=\boldsymbol{\Delta}_{+}^{-1}=\left(\begin{array}{cccccc}
0 & 0 & 0 & \cdots & 0 & 1 \\
1 & 0 & 0 & \cdots & 0 & 0 \\
0 & 1 & 0 & \cdots & 0 & 0 \\
0 & 0 & 1 & \cdots & 0 & 0 \\
\vdots & \vdots & \vdots & \ddots & \vdots & \vdots \\
0 & 0 & 0 & \cdots & 1 & 0
\end{array}\right)
$$

namely, in components

$$
\left(\boldsymbol{\Delta}_{+}\right)_{r s}=\delta_{r, s-1}, \quad\left(\boldsymbol{\Delta}_{-}\right)_{r s}=\delta_{r, s+1} .
$$

In the continuum the derivative $\partial \varphi$ is just the commutator $[\partial, \varphi]$; on the lattice however the commutator $\left[\boldsymbol{\Delta}_{+}, \varphi\right]$ is not diagonal, its non vanishing matrix elements being on a shifted diagonal as in $\boldsymbol{\Delta}_{+}$. In order to write the finite difference (3.5) as a function defined on the lattice sites, namely a diagonal matrix, we have to define it as:

$$
\left(\partial_{+} \boldsymbol{\varphi}\right)=\boldsymbol{\Delta}_{-}\left[\boldsymbol{\Delta}_{+}, \boldsymbol{\varphi}\right]=\left(\begin{array}{cccccc}
\varphi_{1}-\varphi_{N} & 0 & 0 & 0 & \cdots & 0 \\
0 & \varphi_{2}-\varphi_{1} & 0 & 0 & \cdots & 0 \\
0 & 0 & \varphi_{3}-\varphi_{2} & 0 & \cdots & 0 \\
\vdots & \vdots & \vdots & \vdots & \ddots & \vdots \\
0 & 0 & 0 & 0 & \cdots & \varphi_{N}-\varphi_{N-1}
\end{array}\right)
$$


The factor $\boldsymbol{\Delta}_{-}$in front of the commutator is responsible for the violation of the Leibniz rule, in fact from the definition (3.9) we have:

$$
\left(\partial_{+} \boldsymbol{\varphi} \psi\right)=\left(\partial_{+} \boldsymbol{\varphi}\right) \boldsymbol{\psi}+\boldsymbol{\Delta}_{-} \boldsymbol{\varphi} \boldsymbol{\Delta}_{+}\left(\partial_{+} \psi\right)
$$

which is completely equivalent to (3.6). Notice that $\boldsymbol{\Delta}_{-} \boldsymbol{\varphi} \boldsymbol{\Delta}_{+}$is a "shifted" field, where the eigenvalue $\varphi_{r}$ has been replaced by $\varphi_{r-1}:\left(\boldsymbol{\Delta}_{-} \boldsymbol{\varphi}_{\boldsymbol{\Delta}_{+}}\right)_{r}=\boldsymbol{\varphi}_{r-1}$. The correspondence between derivative and finite difference is not one-to-one. In fact the finite difference can be defined as a right or a left difference, so that the following correspondence also holds:

$$
\frac{\partial \varphi(x)}{\partial x} \rightarrow-\frac{1}{a} \partial_{-} \varphi(\boldsymbol{x}) \equiv-\frac{1}{a}(\varphi(\boldsymbol{x})-\varphi(\boldsymbol{x}+a))
$$

The finite difference $\left(\partial_{-} \varphi\right)$ is defined in terms of the shift matrices as

$$
\left(\partial_{-} \varphi\right)=\Delta_{+}\left[\Delta_{-}, \varphi\right]
$$

and satisfies the modified Leibniz rule:

$$
\left(\partial_{-} \boldsymbol{\varphi} \psi\right)=\left(\partial_{-} \boldsymbol{\varphi}\right) \boldsymbol{\psi}+\boldsymbol{\Delta}_{+} \boldsymbol{\varphi} \Delta_{-}\left(\partial_{-} \psi\right)
$$

which shows that $\partial_{-}$carries the same shift as $\partial_{+}$but in the opposite direction. The modified Leibniz rule (3.10) reflects the fact that translational symmetry on the lattice is a discrete, and not a continuous symmetry. To make this point clear consider an action given as the trace of a product of fields $\varphi_{i}$ :

$$
S=\operatorname{Tr} \boldsymbol{\varphi}_{1} \boldsymbol{\varphi}_{2} \cdots \boldsymbol{\varphi}_{r}
$$

The trace corresponds to the sum over all lattice sites, and translational invariance can be simply expressed as the invariance of the trace under cyclic permutations of the eigenvalues, namely (3.14) under

$$
\boldsymbol{\varphi}_{i} \rightarrow \Delta_{-} \boldsymbol{\varphi}_{i} \Delta_{+}=\boldsymbol{\varphi}_{i}+\delta \boldsymbol{\varphi}_{i}
$$

where $\delta \boldsymbol{\varphi}_{i}=-\left(\partial_{+} \boldsymbol{\varphi}\right)$ as defined in (3.9). When the r.h.s. of (3.15) is inserted into (3.14) all orders of $\delta \boldsymbol{\varphi}_{i}$ must be kept in order to preserve the exact symmetry and the variation of the Lagrangian can be cast in the form:

$$
\begin{aligned}
& \delta\left(\boldsymbol{\varphi}_{1} \boldsymbol{\varphi}_{2} \cdots \boldsymbol{\varphi}_{r}\right)=\left(\delta \boldsymbol{\varphi}_{1}\right) \boldsymbol{\varphi}_{2} \cdots \boldsymbol{\varphi}_{r-1} \boldsymbol{\varphi}_{r}+\left(\boldsymbol{\varphi}_{1}+\delta \boldsymbol{\varphi}_{1}\right)\left(\delta \boldsymbol{\varphi}_{2}\right) \boldsymbol{\varphi}_{3} \cdots \boldsymbol{\varphi}_{r}+\cdots \\
& +\left(\boldsymbol{\varphi}_{1}+\delta \boldsymbol{\varphi}_{1}\right)\left(\boldsymbol{\varphi}_{2}+\delta \boldsymbol{\varphi}_{2}\right) \cdots\left(\boldsymbol{\varphi}_{r-1}+\delta \boldsymbol{\varphi}_{r-1}\right)\left(\delta \boldsymbol{\varphi}_{r}\right)
\end{aligned}
$$

which is again the modified Leibniz rule. It is clear that eq. (3.16) follows directly from the invariance of (3.14) under (3.15) by keeping all orders in $\delta \boldsymbol{\varphi}_{i}$ while linear terms in $\delta \boldsymbol{\varphi}_{i}$ give the ordinary Leibniz rule typical of the continuum limit. We stressed this point because the situation is different in the supersymmetric theory discussed in the following sections: supersymmetry charges are non diagonal and hence supersymmetry transformations of a product of superfields obey a modified Leibniz rule on the lattice [5], however these modified Leibniz rules cannot be derived, at least in the present formulation, from a field transformation as in (3.15).

In the approach of ref. [5], which we follow here, in order to preserve all supersymmetries exactly on the lattice a shift is associated to each (super)symmetry charge. Such shifts generate the extended lattice required to describe supersymmetric theories and each shift coincides with the one appearing in the corresponding modified Leibniz rule. 


\section{The $N=2$ one dimensional supersymmetric model on the lattice}

\subsection{The algebra}

The modified Leibniz rule discussed in the previous section for the non supersymmetric case is a key ingredient in the formulation of lattice supersymmetry of ref. [5], which we will follow. In this formulation a shift is associated to each (super)charge, and appears in the corresponding modified Leibniz rule. So, if $\delta_{\alpha}$ is the supersymmetry variation generated by the SUSY charge $Q_{\alpha}$ and and $\Phi_{i}(\theta, x)(i=1,2)$ are superfields, the shift $a_{\alpha}$ associated to $Q_{\alpha}$ will determine the modified Leibniz rule:

$$
\delta_{\alpha}\left(\Phi_{1}(\theta, x) \Phi_{2}(\theta, x)\right)=\left(\delta_{\alpha} \Phi_{1}(\theta, x)\right) \Phi_{2}(\theta, x)+\Phi_{1}\left(\theta, x+a_{\alpha}\right)\left(\delta_{\alpha} \Phi_{2}(\theta, x)\right)
$$

The shifts generate the lattice on which the theory is defined, very much in the same way as roots generate the root lattice. In the supersymmetric case this lattice will have in general extra points and extra links with respect to the bosonic case, the extra links corresponding to the shifts of the supersymmetry charges. As in the case of the roots, non vanishing (anti)commutators give rise to linear constraints among the shifts as required by consistency with (4.1). So, if for instance $\left\{Q_{\alpha}, Q_{\beta}\right\}=P_{\mu}$ and we apply both sides of the equation to a superfield product using the modified Leibniz rule (4.1) we obtain $a_{\alpha}+a_{\beta}= \pm n_{\mu}$, where $n_{\mu}$ is the shift corresponding to a link in the $\mu$ direction and the sign ambiguity is related to the fact that on the lattice $P_{\mu}$ may be represented by either $\boldsymbol{\Delta}_{+}$or $\boldsymbol{\Delta}_{-}$as discussed in the previous section.

The one dimensional supersymmetric model discussed in section 2 has two supersymmetry charges $Q_{1}$ and $Q_{2}$ satisfying the algebra (2.1), notably $Q_{1}^{2}=Q_{2}^{2}=P_{x}$. If we denote by $a_{Q_{1}}$, $a_{Q_{2}}$ and $a_{P_{x}}$ the shifts associated respectively to $Q_{1}, Q_{2}$ and $P_{x}$ we have:

$$
2 a_{Q_{1}}= \pm a_{P_{x}}, \quad 2 a_{Q_{2}}= \pm a_{P_{x}} .
$$

Since $2\left|a_{Q_{i}}\right|=\left|a_{P_{x}}\right|$ it is convenient to use $\left|a_{Q_{i}}\right|$ as lattice spacing $a=\left|a_{Q_{i}}\right|$ and associate to the derivative $\frac{\partial}{\partial x}$ a finite difference of two lattice spacings, namely in the matrix notation:

$$
\frac{\partial}{\partial x} \rightarrow \pm \frac{1}{2 a} \Delta_{ \pm}^{2}
$$

where the dimension $N$ of the matrix, namely the number of sites in the lattice will then be assumed to be even. As for the signs in eq. (4.2) there are essentially two possible distinct choices, namely $a_{Q_{1}}$ and $a_{Q_{2}}$ equal or opposite. Both choices are consistent from the point of view of the realization of SUSY algebra on the lattice, we will choose $a_{Q_{1}}=-a_{Q_{2}}$ which is the most symmetric with respect to the reflection symmetry. The non vanishing commutators of the algebra (2.1) are replaced on the lattice by:

$$
Q_{1}^{2}=-\frac{1}{2 a} \boldsymbol{\Delta}_{-}^{2}, \quad Q_{2}^{2}=\frac{1}{2 a} \boldsymbol{\Delta}_{+}^{2}
$$

and the representation of the supersymmetry charges in terms of the Grassmann coordinates $\theta_{i}$ by:

$$
Q_{1}=\frac{\partial}{\partial \theta_{1}}-\frac{1}{2 a} \theta_{1} \boldsymbol{\Delta}_{-}^{2}, \quad Q_{2}=\frac{\partial}{\partial \theta_{2}}+\frac{1}{2 a} \theta_{2} \boldsymbol{\Delta}_{+}^{2}
$$

Although formally written in the same way as in the continuum case, $\theta_{i}$ and $\frac{\partial}{\partial \theta_{i}}$ in eq. (4.5) are not simply anticommuting parameters and differential operators. In fact, for the supersymmetry 
charges $Q_{i}$ in (4.5) to satisfy the modified Leibniz rules (4.1), it is necessary that $\theta_{i}$ and $\frac{\partial}{\partial \theta_{i}}$ themselves carry a shift. This can be easily determined by requiring that all terms in each of eq.s (4.5) carry the same shift. The result is that $\theta_{1}$ must contain a shift factor $\boldsymbol{\Delta}_{+}$and $\theta_{2}$ a shift factor $\boldsymbol{\Delta}_{-}$(the opposite for the $\frac{\partial}{\partial \theta_{i}}$ ). It is convenient then to represent $\theta_{i}$ as $4 N \times 4 N$ matrices acting on a space that is the direct product of the $N$ dimensional space of the lattice sites and of an internal 4 dimensional space, which is in turn a direct product of two two-dimensional spaces in which $\theta_{i}$ are represented essentially by Pauli matrices:

$$
\begin{aligned}
\theta_{1} & \equiv L^{1 / 2} \sigma_{+} \otimes \mathbf{1} \otimes \Delta_{+}, & \theta_{2} & \equiv L^{1 / 2} \sigma_{3} \otimes \sigma_{+} \otimes \Delta_{-}, \\
\frac{\partial}{\partial \theta_{1}} & \equiv L^{-1 / 2} \sigma_{-} \otimes \mathbf{1} \otimes \Delta_{-}, & \frac{\partial}{\partial \theta_{2}} & \equiv L^{-1 / 2} \sigma_{3} \otimes \sigma_{-} \otimes \Delta_{+} ;
\end{aligned}
$$

where $L$ is the length of the lattice as defined in (3.1).

Eq.s (4.6) and (4.7) can be written explicitly as:

$$
\begin{aligned}
& \theta_{1} \equiv L^{1 / 2}\left(\begin{array}{cccc}
0 & 0 & \Delta_{+} & 0 \\
0 & 0 & 0 & \Delta_{+} \\
0 & 0 & 0 & 0 \\
0 & 0 & 0 & 0
\end{array}\right), \theta_{2} \equiv L^{1 / 2}\left(\begin{array}{cccc}
0 & \Delta_{-} & 0 & 0 \\
0 & 0 & 0 & 0 \\
0 & 0 & 0 & -\Delta_{-} \\
0 & 0 & 0 & 0
\end{array}\right), \\
& \frac{\partial}{\partial \theta_{1}} \equiv L^{-1 / 2}\left(\begin{array}{cccc}
0 & 0 & 0 & 0 \\
0 & 0 & 0 & 0 \\
\Delta_{-} & 0 & 0 & 0 \\
0 & \Delta_{-} & 0 & 0
\end{array}\right), \quad \frac{\partial}{\partial \theta_{2}} \equiv L^{-1 / 2}\left(\begin{array}{cccc}
0 & 0 & 0 & 0 \\
\Delta_{+} & 0 & 0 & 0 \\
0 & 0 & 0 & 0 \\
0 & 0 & -\Delta_{+} & 0
\end{array}\right),
\end{aligned}
$$

where the entries of the above matrices are $N \times N$ matrices and $\Delta_{+}$and $\Delta_{-}$are the shift matrices defined in (3.7).

It is straightforward to check that the matrices (4.8) and (4.9) satisfy the standard Grassmann algebra of the $\theta$ variables. This matrix representation is quite general and can be easily extended to an arbitrary number $n$ of variables by using direct products of $n$ Pauli matrices, namely $2^{n} \times 2^{n}$ matrices.

The factors $L^{ \pm 1 / 2}$ in (4.6) and (4.7) have been introduced for dimensional reasons. In fact it is clear from (4.4) and (4.5) that the Grassmann variables $\theta_{i}$ have dimensions of a square root of a length. The size $L$ of the lattice is kept fixed in performing the continuum limit $a \rightarrow 0$ which becomes equivalent to the ordinary large $N$ limit in matrix models. All dimensional quantities can be expressed in units of $L$, which can then be consistently set to 1 . This notation will be used, unless otherwise specified, in what follows. In these units the lattice spacing $a$ is replaced by $\frac{1}{N}$ and the supercharges are given by:

$$
Q_{1}=\frac{\partial}{\partial \theta_{1}}-\frac{N}{2} \theta_{1} \Delta_{-}^{2}, \quad Q_{2}=\frac{\partial}{\partial \theta_{2}}+\frac{N}{2} \theta_{2} \Delta_{+}^{2}
$$

and the factors $L^{ \pm 1 / 2}$ in the matrix expressions (4.6), (4.7), (4.8) and (4.9) for $\theta_{i}$ and $\frac{\partial}{\partial \theta_{i}}$ are omitted.

\subsection{Fields and superfields}

The next ingredient we need in order to construct a supersymmetric lattice theory is to introduce fields and superfields. Let us begin with the component fields. As discussed in the previous 
sections a scalar field is represented by a diagonal matrix in the $N$ dimensional lattice space. In order to provide its representation in the $4 N$ dimensional space introduced above we need to use its (anti)commutation properties with $\theta_{i}$ and $\frac{\partial}{\partial \theta_{i}}$ and hence distinguish between bosonic and fermionic fields:

- Bosonic field: a field which commutes with all $\theta$ 's and $\frac{\partial}{\partial \theta}$ 's. A straightforward calculation gives

$$
\hat{\varphi} \equiv\left(\begin{array}{cccc}
\varphi & 0 & 0 & 0 \\
0 & \Delta_{+} \varphi \Delta_{-} & 0 & 0 \\
0 & 0 & \Delta_{-} \varphi \Delta_{+} & 0 \\
0 & 0 & 0 & \varphi
\end{array}\right) \quad \begin{aligned}
\varphi & \equiv N \times N \text { matrix } \\
\theta_{i} \hat{\varphi} & =\hat{\varphi} \theta_{i} \\
\frac{\partial}{\partial \theta_{i}} \hat{\varphi} & =\hat{\varphi} \frac{\partial}{\partial \theta_{i}}
\end{aligned}
$$

- Fermionic field: a field which anticommutes with all $\theta$ 's and $\frac{\partial}{\partial \theta}$ 's. A straightforward calculation gives

$$
\underline{\hat{\psi}} \equiv\left(\begin{array}{cccc}
\frac{\psi}{0} & 0 & 0 & 0 \\
0 & -\Delta_{+} \underline{\psi} \Delta_{-} & 0 & 0 \\
0 & 0 & -\Delta_{-} \underline{\psi} \Delta_{+} & 0 \\
0 & 0 & \underline{\psi}
\end{array}\right) \quad \begin{aligned}
\underline{\psi} \hat{\hat{\psi}} & \equiv N \times N \text { fermionic matrix } \\
\frac{\partial}{\partial \theta_{i}} \underline{\hat{\psi}} & =-\underline{\hat{\psi}} \theta_{i} \frac{\partial}{\partial \theta_{i}}
\end{aligned}
$$

Let us now introduce superfields. They can be defined as objects having the standard expansion in terms of $\theta_{i}$, namely

$$
\Phi=\hat{\varphi}+i \theta_{1} \underline{\psi}_{1}+i \theta_{2} \underline{\psi}_{2}+i \theta_{2} \theta_{1} \hat{D},
$$

or equivalently as a matrix commuting with all $\theta$ 's but not with $\frac{\partial}{\partial \theta}$ 's. Either way we get the following representation:

$$
\Phi=\left(\begin{array}{cccc}
\varphi & -i \underline{\psi}_{2} \Delta_{-} & -i \underline{\psi}_{1} \Delta_{+} & i D \\
0 & \Delta_{+} \Delta_{-} & 0 & i \Delta_{+} \underline{\psi}_{1} \\
0 & 0 & \Delta_{-} \Delta_{+} & -i \Delta_{-} \underline{\psi}_{2} \\
0 & 0 & 0 & \varphi
\end{array}\right)
$$

As discussed in the previous sections a scalar field is represented by a diagonal matrix whose eigenvalues are the value of the field at the $N$ lattice sites. A diagonal matrix has no shift attached to it, so a scalar field carries no shift. We assume then that $\varphi$ in eq. (4.11) is diagonal. In order for the superfield $\Phi$ to obey well defined modified Leibniz rules we are forced to assume that all terms of the expansion at the r.h.s. of (4.13) be homogeneous to $\hat{\varphi}$ and carry no shift. This means that the fermionic fields $\underline{\psi}_{1}$ and $\underline{\psi}_{2}$ must carry shifts opposite to the ones of $\theta_{1}$ and $\theta_{2}$, namely that they must be proportional respectively to $\Delta_{-}$and $\Delta_{+}$. It is convenient then to introduce diagonal matrices $\psi_{1}$ and $\psi_{2}$ (not underlined Greek letters):

$$
\psi_{1}=\Delta_{+} \underline{\psi}_{1}, \quad \psi_{2}=\Delta_{-} \underline{\psi}_{2},
$$

which satisfy the same relations (4.12) as the underlined ones. In the matrix representation (4.14) of the superfield all entries are then diagonal matrices, and its explicit form now reads:

$$
\Phi=\left(\begin{array}{cccc}
\varphi & -i \Delta_{+} \psi_{2} \Delta_{-} & -i \Delta_{-} \psi_{1} \Delta_{+} & i D \\
0 & \Delta_{+} \varphi \Delta_{-} & 0 & i \psi_{1} \\
0 & 0 & \Delta_{-} \Delta_{+} & -i \psi_{2} \\
0 & 0 & 0 & \varphi
\end{array}\right)
$$


This is the form of the superfield we will mostly refer to in what follows.

The last point to investigate is the continuum limit of the bosonic and fermionic fields $\varphi$ and $\psi_{i}$. We will assume that $\varphi$ and any bosonic field has a smooth continuum limit. More precisely we assume that the finite difference $\varphi_{r+1}-\varphi_{r}$ is of order of the lattice spacing $a \sim \frac{1}{N}$ in the continuum limit. This means that the derivative is well defined even if the finite difference is taken on a single lattice spacing. If this is true for any bosonic field, it must be true also for the product of two fermionic fields, for instance $\psi_{1} \psi_{2}:\left(\psi_{1}\right)_{r+1}\left(\psi_{2}\right)_{r+1}-\left(\psi_{1}\right)_{r}\left(\psi_{2}\right)_{r}=O(a)$ $(a \rightarrow 0)$. On the other hand, since the continuum derivative corresponds on the lattice to a finite difference over two lattice spacings, for it to be well defined we only need to impose the smoothness condition $\left(\psi_{i}\right)_{r+2}-\left(\psi_{i}\right)_{r}=O(a)$. These two requirements leave us with two possibilities:

- A) $\psi_{i}$ are themselves smooth matrices, like the bosonic fields:

$$
\Delta_{-} \psi_{i} \Delta_{+}-\psi_{i}=O(a), \quad a \rightarrow 0
$$

- B) $\psi_{i}$ are smooth up to an alternating sign:

$$
\Delta_{-} \psi_{i} \Delta_{+}+\psi_{i}=O(a), \quad a \rightarrow 0 .
$$

In the latter B) case $\psi_{i}$ are not themselves smooth but can be written as

$$
\psi_{i}=\mathbf{T} \psi_{i}^{(c)}, \quad(\text { case B })
$$

where $\psi_{i}^{(c)}$ satisfies the condition (4.17), and $\mathbf{T}$ is the alternating $N \times N$ matrix:

$$
\mathbf{T}=\left(\begin{array}{cccccc}
1 & 0 & 0 & 0 & \cdots & 0 \\
0 & -1 & 0 & 0 & \cdots & 0 \\
0 & 0 & 1 & 0 & \cdots & 0 \\
\vdots & \vdots & \vdots & \vdots & \ddots & \vdots \\
0 & 0 & 0 & 0 & \cdots & -1
\end{array}\right)
$$

The matrix $\mathbf{T}$ anticommutes with $\Delta_{ \pm}$, and hence with $\theta_{i}$ and $\frac{\partial}{\partial \theta_{i}}$. By using (4.19) the matrix representation for the (diagonal) fermionic field $\hat{\psi}$ takes the form:

$$
\hat{\psi}=\hat{\mathbf{T}} \hat{\hat{\psi}}, \quad(\text { case } \mathrm{B})
$$

where

$$
\hat{\hat{\psi}}=\left(\begin{array}{cccc}
\psi^{(c)} & 0 & 0 & 0 \\
0 & \Delta_{+} \psi^{(c)} \Delta_{-} & 0 & 0 \\
0 & 0 & \Delta_{-} \psi^{(c)} \Delta_{+} & 0 \\
0 & 0 & 0 & \psi^{(c)}
\end{array}\right)
$$

and

$$
\hat{\mathbf{T}}=\left(\begin{array}{cccc}
\mathbf{T} & 0 & 0 & 0 \\
0 & \mathbf{T} & 0 & 0 \\
0 & 0 & \mathbf{T} & 0 \\
0 & 0 & 0 & \mathbf{T}
\end{array}\right)
$$


Except for its entries being Grassmann odd $\hat{\hat{\psi}}$ has exactly the same structure as the bosonic field $\hat{\varphi}$ in (4.11) and hence it commutes with $\theta_{i}$ and $\frac{\partial}{\partial \theta_{i}}$. The anticommuting properties of $\hat{\psi}$ with respect to the $\theta$ s comes entirely from the matrix $\hat{\mathbf{T}}$ in (4.21). Something similar happens in case A), where $\psi_{i}$ obeys the smoothness condition (4.17) and hence, setting the convention that the superscript $(c)$ always denotes a smooth matrix, we can write:

$$
\psi=\psi^{(c)}, \quad \text { ( case A ). }
$$

Eq. (4.12) then takes the form, similar to (4.21)

$$
\hat{\psi}=\hat{\mathbf{W}} \hat{\hat{\psi}}, \quad(\text { case } \mathrm{A}),
$$

where

$$
\hat{\mathbf{W}}=\left(\theta_{1} \frac{\partial}{\partial \theta_{1}}-\frac{\partial}{\partial \theta_{1}} \theta_{1}\right)\left(\theta_{2} \frac{\partial}{\partial \theta_{2}}-\frac{\partial}{\partial \theta_{2}} \theta_{2}\right)=\left(\begin{array}{cccc}
\mathbf{1} & 0 & 0 & 0 \\
0 & -\mathbf{1} & 0 & 0 \\
0 & 0 & -\mathbf{1} & 0 \\
0 & 0 & 0 & \mathbf{1}
\end{array}\right)
$$

\subsection{Supercharges and susy transformations}

The two supercharges $Q_{1}$ and $Q_{2}$ were given on the lattice by eq.s (4.10). With the matrix representation for the Grassmann coordinates $\theta_{i}$ introduced in the previous sections they can be written in the form:

$$
\begin{gathered}
Q_{1}=\frac{\partial}{\partial \theta_{1}}-\theta_{1} \frac{N \Delta_{-}^{2}}{2}=\left(\begin{array}{cccc}
0 & 0 & -\frac{N \Delta_{-}}{2} & 0 \\
0 & 0 & 0 & -\frac{N \Delta_{-}}{2} \\
\Delta_{-} & 0 & 0 & 0 \\
0 & \Delta_{-} & 0 & 0
\end{array}\right), \\
Q_{2}=\frac{\partial}{\partial \theta_{2}}+\theta_{2} \frac{N \Delta_{+}^{2}}{2}=\left(\begin{array}{cccc}
0 & \frac{N \Delta_{+}}{2} & 0 & 0 \\
\Delta_{+} & 0 & 0 & 0 \\
0 & 0 & 0 & -\frac{N \Delta_{+}}{2} \\
0 & 0 & -\Delta_{+} & 0
\end{array}\right) .
\end{gathered}
$$

The supersymmetry transformations of the superfield $\Phi$, defined in (4.13) and (4.16), will be defined in the usual way as

$$
\delta_{1} \Phi=\underline{\hat{\eta}}_{1}\left[Q_{1}, \Phi\right], \quad \delta_{2} \Phi=\underline{\hat{\eta}}_{2}\left[Q_{2}, \Phi\right],
$$

where $\underline{\hat{q}}_{i}$ are the superparameters of the transformation that we are going presently to discuss. First we notice that the supercharges $Q_{1}$ and $Q_{2}$ carry a shift as they are proportional respectively to $\Delta_{-}$and to $\Delta_{+}$. In order for $\delta_{i} \Phi$ to be shiftless like $\Phi$ itself it is necessary that the superparameters $\underline{\hat{\eta}}_{i}$ also carry a shift opposite to the one of the corresponding charges. So $\underline{\hat{\eta}}_{1}$ must be proportional to $\Delta_{+}$and $\underline{\hat{\eta}}_{2}$ proportional to $\Delta_{-}$:

$$
\underline{\underline{\eta}}_{1}=\Delta_{+} \hat{\eta}_{1}, \quad \underline{\hat{\eta}}_{2}=\Delta_{-} \hat{\eta}_{2},
$$

where $\hat{\eta}_{i}$ has no shift, namely all its entries are diagonal matrices. As in the case of the fermionic fields discussed in the previous section there are two different possibilities, that we labeled case A) and B), for $\hat{\eta}_{i}$ to anticommute with the $\theta_{i}$. We set:

$$
\hat{\eta}_{i}=\hat{\mathbf{W}} \eta_{i} \quad(\text { caseA }), \quad \hat{\eta}_{\mathrm{i}}=\hat{\mathbf{T}} \eta_{\mathrm{i}} \quad(\text { caseB }),
$$


where $\hat{\mathbf{W}}$ and $\hat{\mathbf{T}}$ are given respectively in eq.s (4.26), (4.20) and (4.23) and $\eta_{i}$ are just c-number Grassmann odd parameters. The supersymmetry transformations (4.28) are now completely determined. By expanding the superfield in $\theta_{i}$ or equivalently by using the matrix representation (4.16) one can write the supersymmetry transformations for each component. Again we have to distinguish the two possible discretizations, A) and B). In the case labeled A) we have for $\delta_{1}$ :

$$
\begin{aligned}
\delta_{1} \varphi & =i \eta_{1} \psi_{1} & \delta_{1} D & =\eta_{1} N \frac{\Delta_{+}^{2}\left[\Delta_{-}^{2}, \psi_{2}\right]}{2} \\
\delta_{1} \psi_{1} & =-i \eta_{1} N \frac{\Delta_{+}^{2}\left[\Delta_{-}^{2}, \varphi\right]}{2} & \delta_{1} \psi_{2} & =\eta_{1} D,
\end{aligned}
$$

and for $\delta_{2}$ we have:

$$
\begin{aligned}
& \delta_{2} \varphi=i \eta_{2} \psi_{2} \quad \delta_{2} D=\eta_{2} N \frac{\Delta_{-}^{2}\left[\Delta_{+}^{2}, \psi_{1}\right]}{2} \\
& \delta_{2} \psi_{1}=-\eta_{2} D \quad \delta_{2} \psi_{2}=i \eta_{2} N \frac{\Delta_{-}^{2}\left[\Delta_{+}^{2}, \varphi\right]}{2},
\end{aligned}
$$

where $\psi_{1}$ and $\psi_{2}$ are the diagonal, smooth matrices defined in $(4.24)^{3}$. The transformations (4.31) and (4.32) coincide with the ones given in (2.8) in the continuum limit. In the case labeled B) the supersymmetry transformations can be written in terms of the smooth matrices $\psi_{i}^{(c)}$ defined in (4.19). The result is the same as the one for the case A) except for a sign change in the susy transformations of the bosonic fields:

$$
\begin{aligned}
\delta_{1} \varphi & =-i \eta_{1} \psi_{1}^{(c)} & \delta_{1} D & =-\eta_{1} N \frac{\Delta_{+}^{2}\left[\Delta_{-}^{2}, \psi_{2}^{(c)}\right]}{2} \\
\delta_{1} \psi_{1}^{(c)} & =-i \eta_{1} N \frac{\Delta_{+}^{2}\left[\Delta_{-}^{2}, \varphi\right]}{2} & \delta_{1} \psi_{2}^{(c)} & =\eta_{1} D,
\end{aligned}
$$

and:

$$
\begin{aligned}
\delta_{2} \varphi & =-i \eta_{2} \psi_{2}^{(c)} & \delta_{2} D & =-\eta_{2} N \frac{\Delta_{-}^{2}\left[\Delta_{+}^{2}, \psi_{1}^{(c)}\right]}{2} \\
\delta_{2} \psi_{1}^{(c)} & =-\eta_{2} D & \delta_{2} \psi_{2}^{(c)} & =i \eta_{2} N \frac{\Delta_{-}^{2}\left[\Delta_{+}^{2}, \varphi\right]}{2} .
\end{aligned}
$$

It can be easily verified that these susy transformations form a closed algebra. For instance if

$$
\delta_{2} \Phi=\hat{\eta}_{2} \Delta_{-}\left[Q_{2}, \Phi\right], \quad \delta_{2}^{\prime} \Phi=\hat{\eta}_{2}^{\prime} \Delta_{-}\left[Q_{2}, \Phi\right]
$$

we have

$$
\left(\delta_{2} \delta_{2}^{\prime}-\delta_{2}^{\prime} \delta_{2}\right) \Phi=2 \eta_{2}^{\prime} \eta_{2} \Delta_{-}^{2}\left\{Q_{2},\left[Q_{2} \Phi\right]\right\}=2 \eta_{2}^{\prime} \eta_{2} \Delta_{-}^{2}\left[\frac{N \hat{\Delta}_{+}^{2}}{2}, \Phi\right]
$$

where with a rather obvious notation $\hat{\Delta}_{+}$is a $4 \times 4$ diagonal block matrix whose diagonal entries are $\Delta_{+}$. Likewise for $\delta_{1}$ :

$$
\left(\delta_{1} \delta_{1}^{\prime}-\delta_{1}^{\prime} \delta_{1}\right) \Phi=2 \eta_{1}^{\prime} \eta_{1} \Delta_{+}^{2}\left[-\frac{N \hat{\Delta}_{-}^{2}}{2}, \Phi\right]
$$

\footnotetext{
${ }^{3}$ We also remind the reader that all dimensional quantities are expressed in units of the lattice length $L$, so that the lattice spacing $a$ is just in these units $a=\frac{1}{N}$.
} 
and

$$
\left(\delta_{1} \delta_{2}-\delta_{2} \delta_{1}\right) \Phi=0 .
$$

The superparameters $\underline{\hat{\eta}}_{i}$ of the supersymmetry transformations (4.28) carry a shift (4.29), hence the susy transformation of a product of superfields satisfies a modified Leibniz rule. For instance, if we consider the variation under $Q_{1}$ we have:

$$
\delta_{1}\left(\Phi_{1} \Phi_{2}\right)=\hat{\eta}_{1} \Delta_{+}\left[Q_{1}, \Phi_{1} \Phi_{2}\right]=\left(\delta_{1} \Phi_{1}\right) \Phi_{2}+\left(\Delta_{+} \Phi_{1} \Delta_{-}\right) \delta_{1} \Phi_{2}
$$

where $\Delta_{+} \Phi_{1} \Delta_{-}$is a shifted superfield, namely a superfield where all eigenvalues of the diagonal matrices within $\Phi_{1}$ have been shifted of one unit. A similar expression can be obtained for variations under $Q_{2}$. Supersymmetry transformations of products of an arbitrary number of superfields are uniquely and unambiguously determined by eq. (4.39). However, when using modified Leibniz rules on the component transformations, like for instance (4.31), some care has to be used. In fact on the lattice, due to the shift carried by the $\theta_{i}$, superfields do not commute:

$$
\Phi_{1} \Phi_{2} \neq \Phi_{2} \Phi_{1}
$$

Nevertheless their first components, being diagonal matrices, do commute. So, if we denote by $\left.\Phi\right|_{0} \equiv \hat{\varphi}$ the first component of the superfield $\Phi$ in the $\theta$ expansion, namely its diagonal part in the matrix representation of eq. (4.11), we have

$$
\left.\left(\Phi_{1} \Phi_{2}\right)\right|_{0}=\left.\left(\Phi_{2} \Phi_{1}\right)\right|_{0}
$$

that is

$$
\hat{\varphi}^{(1)} \hat{\varphi}^{(2)}=\hat{\varphi}^{(2)} \hat{\varphi}^{(1)} .
$$

However if we compute susy transformations of $\varphi^{(1)} \varphi^{(2)}$ by using the modified Leibniz rules and the component transformations (4.31) we find

$$
\delta_{1}\left(\varphi^{(1)} \varphi^{(2)}\right)=\delta_{1} \varphi^{(1)} \varphi^{(2)}+\Delta_{+} \varphi^{(1)} \Delta_{-} \delta_{1} \varphi^{(2)}=i \eta_{1}\left(\psi_{1}^{(1)} \varphi^{(2)}+\Delta_{+} \varphi^{(1)} \Delta_{-} \psi_{1}^{(2)}\right)
$$

whereas if we start from $\varphi^{(2)} \varphi^{(1)}$ we find:

$$
\delta_{1}\left(\varphi^{(2)} \varphi^{(1)}\right)=\delta_{1} \varphi^{(2)} \varphi^{(1)}+\Delta_{+} \varphi^{(2)} \Delta_{-} \delta_{1} \varphi^{(1)}=i \eta_{1}\left(\psi_{1}^{(2)} \varphi^{(1)}+\Delta_{+} \varphi^{(2)} \Delta_{-} \psi_{1}^{(1)}\right)
$$

In spite of (4.42) the r.h.s. of (4.43) and (4.44) are different because the shifts act on different fields, although of course they would coincide in the continuum limit. In ref. [23] this was presented as an intrinsic ambiguity and inconsistency of the formulation, but it is not so. In fact $\hat{\varphi}^{(1)} \hat{\varphi}^{(2)}=\hat{\varphi}^{(2)} \hat{\varphi}^{(1)}$ can be regarded as the first component of two distinct superfields $\Phi_{1} \Phi_{2}$ and $\Phi_{2} \Phi_{1}$. In the former case $\left(\hat{\varphi}^{(1)} \hat{\varphi}^{(2)}=\left.\left(\Phi_{1} \Phi_{2}\right)\right|_{0}\right)$ eq. (4.43) should be used, in the latter instead $\left(\hat{\varphi}^{(1)} \hat{\varphi}^{(2)}=\left.\left(\Phi_{2} \Phi_{1}\right)\right|_{0}\right)$ one should use eq. (4.44). In any supersymmetric expression, like for instance the action, it is always possible to determine from the higher components to which superfield a particular product of component fields belongs to, so no ambiguity ever arises. Of course this problem can be avoided from the very beginning by using consistently the superfield formalism. 


\section{The action}

We want now to construct an action on the lattice that reproduces eq.s (2.12) and (2.14) in the continuum limit and is invariant under the discrete susy transformations defined in (4.28). Hereafter we concentrate on the case A) in (4.30), and the modification to the case B) is straightforward. To begin with let us introduce the covariant derivatives $\mathcal{D}_{1}$ and $\mathcal{D}_{2}$, defined as:

$$
\begin{gathered}
\mathcal{D}_{1}=\frac{\partial}{\partial \theta_{1}}+\theta_{1} N \frac{\Delta_{-}^{2}}{2}=\left(\begin{array}{cccc}
0 & 0 & N \frac{\Delta_{-}}{2} & 0 \\
0 & 0 & 0 & N \frac{\Delta_{-}}{2} \\
\Delta_{-} & 0 & 0 & 0 \\
0 & \Delta_{-} & 0 & 0
\end{array}\right) \\
\mathcal{D}_{2}=\frac{\partial}{\partial \theta_{2}}-\theta_{2} N \frac{\Delta_{+}^{2}}{2}=\left(\begin{array}{cccc}
0 & -N \frac{\Delta_{+}}{2} & 0 & 0 \\
\Delta_{+} & 0 & 0 & 0 \\
0 & 0 & 0 & N \frac{\Delta_{+}}{2} \\
0 & 0 & -\Delta_{+} & 0
\end{array}\right) .
\end{gathered}
$$

It is straightforward matrix algebra to check that these covariant derivatives anticommute with each other and with all supercharges

$$
\left\{\mathcal{D}_{i}, Q_{j}\right\}=0 \quad \forall i, j \quad \text { and also } \quad\left\{\mathcal{D}_{1}, \mathcal{D}_{2}\right\}=0 .
$$

Besides we have

$$
\mathcal{D}_{1}^{2}=+N \frac{\hat{\Delta}_{-}^{2}}{2}, \quad \mathcal{D}_{2}^{2}=-N \frac{\hat{\Delta}_{+}^{2}}{2} .
$$

The superfield action (2.12) in the continuum can be written on the lattice in the same way, by simply replacing each symbol with the corresponding matrices and the integral over $x$ with the trace:

$$
S=\operatorname{Tr}\left(\left\{\frac{\partial}{\partial \theta_{1}}, \quad\left[\frac{\partial}{\partial \theta_{2}}, \frac{1}{2}\left[\mathcal{D}_{2}, \Phi\right]\left[\mathcal{D}_{1}, \Phi\right]+\mathrm{i} V(\Phi)\right]\right\}\right) .
$$

The invariance of $S$ under supersymmetry transformations on the lattice can easily be proved. First we notice that $\frac{\partial}{\partial \theta_{1}}$ and $\frac{\partial}{\partial \theta_{2}}$ in (5.4) can be replaced with respectively $Q_{1}$ and $Q_{2}$ without affecting the trace, the extra terms being total differences. So we can write:

$$
S=\operatorname{Tr}\left(\left\{Q_{1}, \quad\left[Q_{2}, \frac{1}{2} \Psi_{2} \Psi_{1}+\mathrm{i} V(\Phi)\right]\right\}\right)
$$

where we have defined the fermionic superfields

$$
\Psi_{1}=\left[\mathcal{D}_{1}, \Phi\right], \quad \Psi_{2}=\left[\mathcal{D}_{2}, \Phi\right] .
$$

Consider now the variation $\delta_{1} S$ of the action defined according to eq. (4.28) and (4.29):

$$
\delta_{1} S=\operatorname{Tr}\left(\left\{Q_{1},\left[Q_{2}, \hat{\eta}_{1} \Delta_{+}\left[Q_{1}, \frac{1}{2} \Psi_{2} \Psi_{1}+\mathrm{i} V(\Phi)\right]\right]\right\}\right)
$$

By using Jacobi identities and eq.s (4.4) it is easily seen that the expression under trace in (5.7) is a total difference and hence it vanishes when the trace is taken. The invariance of the action is then proved in complete generality. 
The lattice superfield action (5.4) can be expanded and written in terms of component fields, by using the explicit matrix expression (4.16) of the superfield $\Phi$. Let us consider first only the kinetic term, but including the mass term (that is we set $g=0$ in the expression (2.13)). The action written in terms of component fields then reads:

$$
\begin{aligned}
S= & \operatorname{Tr} \frac{1}{2}\left\{-\left[N \frac{\Delta_{+}^{2}}{2}, \varphi\right]\left[N \frac{\Delta_{-}^{2}}{2}, \varphi\right]-D^{2}+\Delta_{-}^{2}\left[N \frac{\Delta_{+}^{2}}{2}, \psi_{1}\right] \Delta_{-} \psi_{1} \Delta_{+}+\Delta_{+} \psi_{2} \Delta_{-} \Delta_{+}^{2}\left[N \frac{\Delta_{-}^{2}}{2}, \psi_{2}\right]\right\} \\
& -\frac{m}{2} \operatorname{Tr}\left(\varphi D+D \varphi-i \Delta_{+} \psi_{2} \Delta_{-} \psi_{1}+i \Delta_{-} \psi_{1} \Delta_{+} \psi_{2}\right) .
\end{aligned}
$$

In the continuum limit the action (5.8) coincides with the one given in (2.14) with $g=0$. The invariance of (5.8) under susy transformations can be checked by using the component fields transformations (4.31) and (4.32) and the modified Leibniz rule. In agreement with the discussion at the end of the last section, the order of the factors in each term of (5.8) is important in applying the modified Leibniz rule even if the factors (anti)commute, and in fact the order in (5.8) reflect the one that each field had in the original superfield formulation (5.4). Also on the lattice the auxiliary field $D$ can be eliminated by a gaussian integration leading to the action:

$$
\begin{aligned}
S= & \operatorname{Tr} \frac{1}{2}\left\{-\left[N \frac{\Delta_{+}^{2}}{2}, \varphi\right]\left[N \frac{\Delta_{-}^{2}}{2}, \varphi\right]+m^{2} \varphi^{2}+\Delta_{-}^{2}\left[N \frac{\Delta_{+}^{2}}{2}, \psi_{1}\right] \Delta_{-} \psi_{1} \Delta_{+}+\Delta_{+} \psi_{2} \Delta_{-} \Delta_{+}^{2}\left[N \frac{\Delta_{-}^{2}}{2}, \psi_{2}\right]\right\} \\
& -\frac{m}{2} \operatorname{Tr}\left(-i \Delta_{+} \psi_{2} \Delta_{-} \psi_{1}+i \Delta_{-} \psi_{1} \Delta_{+} \psi_{2}\right),
\end{aligned}
$$

which reduces to $(2.15)$ (with $g=0$ ) in the continuum limit.

Let us introduce again the coordinates $\boldsymbol{x}=r a=\frac{r}{N}(r=1,2, \cdots, N)$ on the lattice and represent the diagonal matrices in (5.8) by means on their eigenvalues, using the notation of eq. (3.2). The trace becomes a sum over the coordinates $\boldsymbol{x}$, and the action can be written as:

$$
\begin{aligned}
S= & \sum_{\boldsymbol{x}} \frac{1}{2}\left\{-\frac{\varphi(\boldsymbol{x}+2 a) \varphi(\boldsymbol{x})+\varphi(\boldsymbol{x}) \varphi(\boldsymbol{x}+2 a)-2 \varphi(\boldsymbol{x}) \varphi(\boldsymbol{x})}{4 a^{2}}\right. \\
& +\frac{\psi_{1}(\boldsymbol{x}+a) \psi_{1}(\boldsymbol{x})-\psi_{1}(\boldsymbol{x}) \psi_{1}(\boldsymbol{x}+a)+\psi_{2}(\boldsymbol{x}+a) \psi_{2}(\boldsymbol{x})-\psi_{2}(\boldsymbol{x}) \psi_{2}(\boldsymbol{x}+a)}{2 a}-D(\boldsymbol{x}) D(\boldsymbol{x}) \\
& \left.-m\left[\varphi(\boldsymbol{x}) D(\boldsymbol{x})+D(\boldsymbol{x}) \varphi(\boldsymbol{x})-i \psi_{2}(\boldsymbol{x}+a) \psi_{1}(\boldsymbol{x})+i \psi_{1}(\boldsymbol{x}) \psi_{2}(\boldsymbol{x}+a)\right]\right\} .
\end{aligned}
$$

As in (5.8), the order of the factors in each term has been preserved and it reflects the original order of the superfield product. In this way the action is invariant under supersymmetry transformations, using the modified Leibniz rule whenever susy transformations are applied to products of fields. In the coordinate notation of (5.10) the supersymmetry transformations (4.31) and (4.32) and the modified Leibniz rules are:

$$
\begin{array}{cc}
\delta_{1} \varphi(\boldsymbol{x})=i \eta_{1} \psi_{1}(\boldsymbol{x}) & \delta_{1} D(\boldsymbol{x})=-\eta_{1} \frac{\psi_{2}(\boldsymbol{x}+2 a)-\psi_{2}}{2 a} \\
\delta_{1} \psi_{1}(\boldsymbol{x})=i \eta_{1} \frac{\varphi(\boldsymbol{x}+2 a)-\varphi(\boldsymbol{x})}{2 a} & \delta_{1} \psi_{2}(\boldsymbol{x})=\eta_{1} D(\boldsymbol{x}), \\
\delta_{2} \varphi(\boldsymbol{x})=i \eta_{2} \psi_{2}(\boldsymbol{x}) & \delta_{2} D(\boldsymbol{x})=\eta_{2} \frac{\psi_{1}(\boldsymbol{x})-\psi_{1}(\boldsymbol{x}-2 a)}{2 a} \\
\delta_{2} \psi_{1}(\boldsymbol{x})=-\eta_{2} D(\boldsymbol{x}) & \delta_{2} \psi_{2}(\boldsymbol{x})=i \eta_{2} \frac{\varphi(\boldsymbol{x})-\varphi(\boldsymbol{x}-2 a)}{2 a}
\end{array}
$$


and

$$
\begin{aligned}
& \delta_{1}(A(\boldsymbol{x}) B(\boldsymbol{x}))=\left(\delta_{1} A(\boldsymbol{x})\right) B(\boldsymbol{x})+A(\boldsymbol{x}+a)\left(\delta_{1} B(\boldsymbol{x})\right), \\
& \delta_{2}(A(\boldsymbol{x}) B(\boldsymbol{x}))=\left(\delta_{2} A(\boldsymbol{x})\right) B(\boldsymbol{x})+A(\boldsymbol{x}-a)\left(\delta_{2} B(\boldsymbol{x})\right) .
\end{aligned}
$$

In writing the action and the supersymmetry transformations above we have used the case A), namely we have chosen the fermion matrix fields $\psi_{1}$ and $\psi_{2}$ to have a smooth continuum limit. In case B) a change of sign of the fermionic fields on odd sites is required to obtain a smooth field and performing the continuum limit. The result in the action (5.10) would be a sign change in front of the fermionic action which is not significant and does not need to be discussed any

further. Correspondingly, as already reported in the previous section, a change of sign would occur in the susy transformations of the fermionic fields in (5.11) and (5.12). The order of the different factors arising from the superfield expansion corresponds to a symmetrization of the action, as it is apparent from (5.10). This is needed not just for the invariance of the action under (5.11) and (5.12) with the modified Leibniz rule (5.13), but also for the Hamiltonian to be a self-adjoint operator and hence the propagator to be real. Reflection positivity is also explicitly satisfied by (5.10).

The interaction term can also be calculated from the superfield expansion, and in the same notation of (5.10) is given by:

$$
\begin{aligned}
S_{i n t} & =\frac{g}{4}\left\{-\varphi^{3}(\boldsymbol{x}) D(\boldsymbol{x})-\varphi^{2}(\boldsymbol{x}) D(\boldsymbol{x}) \varphi(\boldsymbol{x})-\varphi(\boldsymbol{x}) D(\boldsymbol{x}) \varphi^{2}(\boldsymbol{x})-D(\boldsymbol{x}) \varphi^{3}(\boldsymbol{x})\right. \\
& +i \varphi^{2}(\boldsymbol{x}) \psi_{2}(\boldsymbol{x}+a) \psi_{1}(\boldsymbol{x})+i \varphi(\boldsymbol{x}) \psi_{2}(\boldsymbol{x}+a) \varphi(\boldsymbol{x}+a) \psi_{1}(\boldsymbol{x})+i \psi_{2}(\boldsymbol{x}+a) \varphi^{2}(\boldsymbol{x}+a) \psi_{1}(\boldsymbol{x}) \\
& +i \psi_{2}(\boldsymbol{x}+a) \varphi(\boldsymbol{x}+a) \psi_{1}(\boldsymbol{x}) \varphi(\boldsymbol{x})+i \psi_{2}(\boldsymbol{x}+a) \psi_{1}(\boldsymbol{x}) \varphi^{2}(\boldsymbol{x})+i \varphi(\boldsymbol{x}) \psi_{2}(\boldsymbol{x}+a) \psi_{1}(\boldsymbol{x}) \varphi(\boldsymbol{x}) \\
& -i \varphi^{2}(\boldsymbol{x}) \psi_{1}(\boldsymbol{x}-a) \psi_{2}(\boldsymbol{x})-i \varphi(\boldsymbol{x}) \psi_{1}(\boldsymbol{x}-a) \varphi(\boldsymbol{x}-a) \psi_{2}(\boldsymbol{x})-i \psi_{1}(\boldsymbol{x}-a) \varphi^{2}(\boldsymbol{x}-a) \psi_{2}(\boldsymbol{x}) \\
& \left.-i \psi_{1}(\boldsymbol{x}-a) \varphi(\boldsymbol{x}-a) \psi_{2}(\boldsymbol{x}) \varphi(\boldsymbol{x})-i \psi_{1}(\boldsymbol{x}-a) \psi_{2}(\boldsymbol{x}) \varphi^{2}(\boldsymbol{x})-i \varphi(\boldsymbol{x}) \psi_{1}(\boldsymbol{x}-a) \psi_{2}(\boldsymbol{x}) \varphi(\boldsymbol{x})\right\} .
\end{aligned}
$$

The interaction term, written in the form (5.14), is also invariant under (5.11), (5.12) and (5.13). All terms in (5.14) can be obtained starting from just two terms, for instance $-\varphi^{3}(\boldsymbol{x}) D(\boldsymbol{x})$ and $i \varphi^{2}(\boldsymbol{x}) \psi_{2}(\boldsymbol{x}+a) \psi_{1}(\boldsymbol{x})$ by means of a symmetrization procedure, that is by taking the factors in all possible orders. However in exchanging two fields the arguments are shifted as if different fields were carrying different shifts, namely 0 for the bosonic fields $\varphi(\boldsymbol{x})$ and $D(\boldsymbol{x})$ and respectively $+a$ and $-a$ for the fermionic field $\psi_{2}(\boldsymbol{x})$ and $\psi_{1}(\boldsymbol{x})$. This symmetrization procedure can be used, in alternative to the superfield expansion and with the same results, to generate the correct susy invariant terms at the component level.

\section{$6 \quad$ Ward identities}

\subsection{Quadratic Ward identities}

The formalism developed in the previous sections has allowed us to construct a one dimensional supersymmetric theory on the lattice with two supersymmetry charges and exact supersymmetry invariance under both of them. Such invariance is in fact satisfied by any model constructed using the superfield (4.16) and the covariant derivatives (5.1) as ingredients. Supersymmetry transformations on the lattice, just as translations, are discrete and, when applied to a product of superfields, obey modified Leibniz rules (4.39). However, unlike translations, where the 
modified Leibniz rule is equivalent to keeping all orders of $\delta \varphi_{i}$ as shown in (3.15) and (3.16), supersymmetry transformations of a product of superfields are not obtained by doing a substitution $\Phi \rightarrow \Phi+\delta_{i} \Phi$ on all terms of the product and keeping all orders of the variation $\delta_{i} \Phi$. This is an important point, as the derivation of Ward identities from a symmetry of the action involves, in the functional integral formalism, a change of variables under which the action and the integration volume are invariant. Such change of variable does not exist for supersymmetry transformations on the lattice, and the standard derivation of the Ward identities is not possible. In this section and in the following one we shall investigate the possibility that correlation functions obey modified Ward identities, defined in a way that is reminiscent of the modified Leibniz rule. This is the case indeed for the free theory (including mass term) to which this section is devoted, whereas for the interacting theory the question is still open.

We begin with considering the quadratic action of eq. (5.10). In order to avoid unnecessary complications we introduce a compact notation and write the action as:

$$
S=\frac{1}{2} \sum_{A, B} f_{A} M_{A B} f_{B}
$$

where the indices $A$ and $B$ are composite indices, namely:

$$
A \equiv(\alpha, x) .
$$

The index $\alpha$ goes from 1 to 4 to denote the four types of component fields $\left\{\varphi, D, \psi_{1}, \psi_{2}\right\}$, while $x$ is just the $N$-valued space time coordinate. We shall also define $|A|$ as two valued function that is 0 (resp. 1) if $f_{A}$ is a bosonic (resp. fermionic) field, so that we have:

$$
f_{A} f_{B}=(-1)^{|A||B|} f_{B} f_{A} .
$$

All the information about the action is contained in the matrix $M_{A B}$, which in agreement with (6.3) can be chosen to satisfy the symmetry:

$$
M_{A B}=(-1)^{|A||B|} M_{B A} .
$$

Another symmetry property of $M_{A B}$ follows from the invariance of the action under translations on the lattice. If we define an operator $T_{+}$(and its inverse $T_{-}$) that acts on the index $A$ shifting the coordinate $x$ of one lattice spacing:

$$
T_{ \pm} A \equiv(\alpha, x \pm a)
$$

then translational invariance implies:

$$
M_{\left(T_{+} A\right)\left(T_{+} B\right)}=M_{A B} .
$$

The supersymmetry transformations (5.11) and (5.12) can be described in this notation by two matrices $\lambda_{A B}^{(i)}(i=1,2)$ and read:

$$
\delta_{i} f_{A}=\eta_{i} \lambda_{A B}^{(i)} f_{B}
$$

The explicit expression of $\lambda_{A B}^{(i)}$ is not important here (it could be easily derived from (5.11) and (5.12)), the only formal property we need is again translational invariance: $\lambda_{A B}^{(i)}=\lambda_{\left(T_{+} A\right)\left(T_{+} B\right)}^{(i)}$; moreover it is worth mentioning that because of its statistic changing nature $\lambda_{A B}^{(i)}$ is non vanishing only for $|A|+|B|=1$. The invariance of the action (6.1) under supersymmetry transformations 
can then be translated into a set of conditions on the matrix $M_{A B}$. In fact by applying the modified Leibniz rule we have:

$$
\delta_{i} S=\sum_{A B}\left(\delta_{i} f_{A}\right) M_{A B} f_{B}+f_{\left(T_{i} A\right)} M_{A B}\left(\delta_{i} f_{B}\right),
$$

where $T_{i}$ is $T_{+}$for $i=1$ and $T_{-}$for $i=2$. By writing the variations explicitly by means of (6.7), and using symmetry properties of $M_{A B}$ and $\lambda_{A B}^{(i)}$ (including translational invariance) one finds that (6.8) is zero iff:

$$
\left(M_{A B}+M_{\left(T_{i} A\right) B}\right) \lambda_{A C}^{(i)}+\left(M_{A C}+M_{\left(T_{i} A\right) C}\right) \lambda_{A B}^{(i)}=0
$$

where the sum over $A$ is understood. One can check that (6.9) is satisfied by (5.10), but it is completely general and depends only by the assumption that the action is quadratic, translationally invariant and invariant under supersymmetry transformations with the modified Leibniz rules.

In order to see how the condition (6.9) can be translated into a relation between correlation functions let us introduce the generating functional of the correlation functions $F(J)$ :

$$
F(J)=\int d f_{A} e^{-\frac{1}{2} f_{A} M_{A B} f_{B}+J_{A} f_{A}}
$$

where the integration volume $d f_{A}$ is normalized so that $F(J=0)=1$. The generating functional $F(J)$ can be calculated explicitly in this case, as the defining integral is quadratic, and is given by:

$$
F(J)=e^{\frac{1}{2} J_{A} M_{A B}^{-1} J_{B}(-1)^{|A|}} .
$$

Correlation functions are obtained by expanding $F(J)$ in powers of $J$. In particular the only connected correlation function of a free theory, the propagator, is given by:

$$
<f_{A} f_{B}>=\left.\frac{\partial}{\partial J_{A}} \frac{\partial}{\partial J_{B}} F(J)\right|_{J=0}=M_{A B}^{-1} .
$$

We can now write (6.9) as a relation among correlation functions by simply multiplying it by $M_{B G}^{-1} M_{C F}^{-1}$ and use again translational invariance. This gives:

$$
\lambda_{G A} M_{A F}^{-1}+\lambda_{G A} M_{A\left(T_{i} F\right)}^{-1}+\lambda_{F A} M_{A G}^{-1}+\lambda_{F A} M_{A\left(T_{i}\right) G}^{-1}=0 .
$$

Replacing in (6.13) $M_{A B}^{-1}$ with the two points correlation function $<f_{A} f_{B}>$ and multiplying by the Grassmann odd parameter of the susy transformation $\eta_{i}$ we finally obtain:

$$
<\left(\delta_{i} f_{G}\right) f_{F}+f_{T_{i} G}\left(\delta_{i} f_{F}\right)+\left(\delta_{i} f_{F}\right) f_{G}+f_{T_{i} F}\left(\delta_{i} f_{G}\right)>=<\delta_{i}\left(f_{G} f_{F}+f_{F} f_{G}\right)>=0,
$$

where the variation of the product in (6.14) is performed using the modified Leibniz rule.

It is interesting to compare the previous result with the one we would obtain by the standard functional integral method, namely by doing in the integral (6.10) the substitution

$$
f_{A} \rightarrow f_{A}+\left(\delta_{i} f_{A}\right)
$$

with $\left(\delta_{i} f_{A}\right)$ given by (6.7). Such substitution however does not leave the action invariant because of the modified Leibniz rule in (6.8), and one obtains:

$$
<e^{J_{A} f_{A}}\left(J_{A}\left(\delta_{i} f_{A}\right)-\delta_{s u b} S\right)>=0,
$$


where $\delta_{\text {sub }} S$ is the variation of the action under the substitution (6.15):

$$
\delta_{s u b} S=\frac{1}{2}\left(f_{B}-f_{\left(T_{i} B\right)}\right) M_{B A}\left(\delta_{i} f_{A}\right)
$$

Notice that $\delta_{s u b} S$, being proportional to $f_{B}-f_{\left(T_{i} B\right)}$ is of order $\frac{1}{N}$ in the large $N$ continuum limit. By expanding (6.16) in $J_{A}$ one obtains all possible Ward identities. In particular, by considering the coefficient of $J_{A} J_{B}$ of the expansion one gets:

$$
<\left(\delta_{i} f_{A}\right) f_{B}+f_{A}\left(\delta_{i} f_{B}\right)-f_{A} f_{B} \delta_{s u b} S>=0 .
$$

The first two terms in (6.18) would give the variation of $f_{A} f_{B}$ if the ordinary Leibniz rule were valid, while the last term is the direct consequence of the non-invariance of the action under the substitution (6.15). This term is quartic in $f_{A}$, and hence it gets contributions from disconnected correlation functions. It is then far from trivial that its effect would be to restore the symmetry by turning the ordinary Leibniz rule into the modified one of the "correct" Ward identities of eq. (6.14).

The existence of modified Ward identities reflects a symmetry of the generating functional of the correlation functions $F(J)$. In fact the exponent at the r.h.s. of (6.11), namely the generator of the connected correlation functions, has the same structure as the quadratic action (but with $M^{-1}$ in place of $M$ ) and is invariant under similar transformations:

$$
\delta_{i} J_{C}=\eta_{i}(-1)^{|C|} J_{F} \lambda_{F C}
$$

provided a modified Leibniz rule (with $T_{i}$ replaced by $T_{i}^{-1}$ ) is applied when taking the variation of a product of sources $J_{A}$. Indeed it is easy to check that in this way the variation of the exponent in (6.11) under (6.19) is proportional to the l.h.s. of (6.13) which is vanishing thanks to the symmetry of the action.

\subsection{Momentum space}

In order to write explicitly the Ward identities associated to the quadratic action (5.10), it is convenient to go to the momentum space and define;

$$
\tilde{f}_{\alpha}(p)=\frac{1}{N} \sum_{r=1}^{N} f_{\alpha}(r) e^{\frac{2 i \pi}{N} p r},
$$

where the integer $r$ and $\alpha$ define together the composite index ${ }^{4} A \equiv\{\alpha, x=a r\}$ used in the previous subsection and

$$
f_{A} \equiv f_{\alpha}(r)
$$

The integer (modulo $N) p$ is the momentum measured in units of $\frac{1}{L}$. The component fields in the momentum space will always be denoted, as in (6.20), with the same letter as the original field surmounted by a tilde. Standard properties of momentum space follow directly from (6.20), for instance the product of two local fields leads to the well known convolution and momentum conservation:

$$
f(x)=f_{1}(x) f_{2}(x) \rightarrow \tilde{f}(p)=\sum_{p_{1}, p_{2}} \tilde{f}_{1}\left(p_{1}\right) \tilde{f}_{2}\left(p_{2}\right) \delta_{p, p_{1}+p_{2}}
$$

\footnotetext{
${ }^{4}$ We remind here that $\alpha=1,2,3,4$ labels the different types of component fields: $f_{\alpha} \equiv\left\{\varphi, D, \psi_{1}, \psi_{2}\right\}$.
} 
All the results of previous sections can be conveniently rephrased in this language. The quadratic action (5.10) for instance can be written as:

$$
\begin{aligned}
S & =\sum_{p=1}^{N}\left\{\frac{N^{2}}{2} \tilde{\varphi}(p) \sin ^{2} \frac{2 \pi}{N} p \tilde{\varphi}(-p)-\frac{i N}{2} \tilde{\psi}_{1}(p) \sin \frac{2 \pi}{N} p \tilde{\psi}_{1}(-p)\right. \\
& -\frac{i N}{2} \tilde{\psi}_{2}(p) \sin \frac{2 \pi}{N} p \tilde{\psi}_{2}(-p)-\frac{1}{2} \tilde{D}(p) \tilde{D}(-p)-\frac{m}{2}[\tilde{\varphi}(p) \tilde{D}(-p)+\tilde{D}(p) \tilde{\varphi}(-p) \\
& \left.\left.-i \omega^{-p} \tilde{\psi}_{2}(p) \tilde{\psi}_{1}(-p)+i \omega^{p} \tilde{\psi}_{1}(p) \tilde{\psi}_{2}(-p)\right]\right\}
\end{aligned}
$$

where $\omega=e^{\frac{2 i \pi}{N}}$. The action $(6.23)$ is invariant under the two sets of supersymmetry transformations; eq.s (4.31) and (4.32), which in the momentum representation read:

$$
\begin{array}{cc}
\delta_{1} \tilde{\varphi}(p)=i \eta_{1} \tilde{\psi}_{1}(p) & \delta_{1} \tilde{D}(p)=i \eta_{1} N \omega^{-p} \sin \frac{2 \pi}{N} p \tilde{\psi}_{2} \\
\delta_{1} \tilde{\psi}_{1}(p)=\eta_{1} N \omega^{-p} \sin \frac{2 \pi}{N} p \tilde{\varphi}(p) & \delta_{1} \tilde{\psi}_{2}(p)=\eta_{1} \tilde{D}(p) \\
\delta_{2} \tilde{\varphi}(p)=i \eta_{2} \tilde{\psi}_{2}(p) & \delta_{2} \tilde{D}(p)=-i \eta_{2} N \omega^{p} \sin \frac{2 \pi}{N} p \tilde{\psi}_{1}(p) \\
\delta_{2} \tilde{\psi}_{1}(p)=-\eta_{2} \tilde{D}(p) & \delta_{2} \tilde{\psi}_{2}(p)=\eta_{2} N \omega^{p} \sin \frac{2 \pi}{N} p \tilde{\varphi}(p) .
\end{array}
$$

When acting on a product of fields the supersymmetry transformations must be applied using the modified Leibniz ${ }^{5}$ rule (5.13), which in the momentum representation are:

$$
\begin{aligned}
& \delta_{1}\left(\tilde{f}_{\alpha}(p) \tilde{f}_{\beta}(q)\right)=\left(\delta_{1} \tilde{f}_{\alpha}(p)\right) \tilde{f}_{\beta}(q)+\omega^{-p} \tilde{f}_{\alpha}(p)\left(\delta_{1} \tilde{f}_{\beta}(q)\right) \\
& \delta_{2}\left(\tilde{f}_{\alpha}(p) \tilde{f}_{\beta}(q)\right)=\left(\delta_{2} \tilde{f}_{\alpha}(p)\right) \tilde{f}_{\beta}(q)+\omega^{p} \tilde{f}_{\alpha}(p)\left(\delta_{2} \tilde{f}_{\beta}(q)\right) .
\end{aligned}
$$

The two points correlation functions can easily be obtained from the action (6.23) and cast in a matrix form:

$$
<\tilde{f}_{\alpha}(q) \tilde{f}_{\beta}(p)>=\delta_{q,-p} M_{\alpha \beta}^{-1}(p)
$$

with

$$
\boldsymbol{M}^{-1}(p)=\frac{1}{N^{2} \sin ^{2} \frac{2 \pi p}{N}+m^{2}}\left(\begin{array}{cccc}
1 & -m & 0 & 0 \\
-m & -N^{2} \sin ^{2} \frac{2 \pi p}{N} & 0 & 0 \\
0 & 0 & i N \sin \frac{2 \pi p}{N} & -i m \omega^{p} \\
0 & 0 & i m \omega^{-p} & i N \sin \frac{2 \pi p}{N}
\end{array}\right)
$$

The argument of $\sin ^{2}$ at the denominator in (6.28) vanishes for two values of $p$, namely $p=0$ and $p=\frac{N}{2}$. This means that there are two poles in the Brillouin zone, namely that the doubling of the fermions is not eliminated in this model but rather generalized to all types of fields to preserve the balance between bosonic and fermionic degrees of freedom. However one can see from (5.10) that odd and even sites are coupled only through the fermionic degrees of freedom, and are decoupled in the bosonic lagrangian. So one set of bosonic degrees of freedom, say the

\footnotetext{
${ }^{5}$ It is worth to remind that the modified Leibniz rule is order sensitive, and that it can be applied to component fields only when the order of the fields reflects the one of the original superfields.
} 
ones on odd sites, could be eliminated. This however would break exact supersymmetry, because bosonic fields on odd sites would be obtained as a result of supersymmetry transformation on odd sites fermions, as well as a result of the modified Leibniz rule.

The supersymmetry transformations (6.24) and (6.25) can also be written in a more compact notation by as

$$
\delta_{1} \tilde{f}_{\alpha}(p)=\eta_{1} \lambda_{\alpha \beta}^{(1)}(p) \tilde{f}_{\beta}(p), \quad \delta_{2} \tilde{f}_{\alpha}(p)=\eta_{2} \lambda_{\alpha \beta}^{(2)}(p) \tilde{f}_{\beta}(p),
$$

where the $4 \times 4$ matrices $\boldsymbol{\lambda}^{(i)}(p)$ are given by:

$$
\begin{aligned}
\lambda^{1}(p) & =\left(\begin{array}{cccc}
0 & 0 & i & 0 \\
0 & 0 & 0 & i N \omega^{-p} \sin \frac{2 \pi p}{N} \\
N \omega^{-p} \sin \frac{2 \pi p}{N} & 0 & 0 & 0 \\
0 & & 0 & 0
\end{array}\right), \\
\lambda^{2}(p) & =\left(\begin{array}{cccc}
0 & 0 & 0 & i \\
0 & 0 & -i N \omega^{p} \sin \frac{2 \pi p}{N} & 0 \\
0 & -1 & 0 & 0 \\
N \omega^{p} \sin \frac{2 \pi p}{N} & 0 & 0 & 0
\end{array}\right)
\end{aligned}
$$

The modified Ward identities (6.14) in the momentum representation take the form:

$$
\delta_{i}<\tilde{f}_{\alpha}(-p) \tilde{f}_{\beta}(p)+\tilde{f}_{\beta}(p) \tilde{f}_{\alpha}(-p)>=0 .
$$

The variation in (6.31) can be performed using eq.s (6.29) and the modified Leibniz rules (6.26), leading to the equations:

$$
\begin{aligned}
& \left(1+\omega^{-p}\right) \lambda_{\alpha \gamma}^{(1)}(-p)<\tilde{f}_{\gamma}(-p) \tilde{f}_{\beta}(p)>+\left(1+\omega^{p}\right) \lambda_{\beta \gamma}^{(1)}(p)<\tilde{f}_{\gamma}(p) \tilde{f}_{\alpha}(-p)>=0 \\
& \left(1+\omega^{p}\right) \lambda_{\alpha \gamma}^{(2)}(-p)<\tilde{f}_{\gamma}(-p) \tilde{f}_{\beta}(p)>+\left(1+\omega^{-p}\right) \lambda_{\beta \gamma}^{(2)}(p)<\tilde{f}_{\gamma}(p) \tilde{f}_{\alpha}(-p)>=0 .
\end{aligned}
$$

These can be written explicitly in terms of the component fields, by assigning specific values to the indices. From the first one, i.e. $\delta_{1}$ variation we get:

$$
\begin{array}{r}
i<\tilde{\psi}_{1}(-p) \tilde{\psi}_{1}(p)>+N \sin \frac{2 \pi p}{N}<\tilde{\varphi}(-p) \tilde{\varphi}(p)>=0 \\
-i N \sin \frac{2 \pi p}{N}<\tilde{\psi}_{2}(-p) \tilde{\psi}_{2}(p)>+<\tilde{D}(-p) \tilde{D}(p)>=0 \\
i<\tilde{\psi}_{1}(-p) \tilde{\psi}_{2}(p)>+\omega^{p}<\tilde{\varphi}(-p) \tilde{D}(p)>=0 .
\end{array}
$$

From the second equation in (6.32), namely the $\delta_{2}$ variation, we get:

$$
\begin{array}{r}
i<\tilde{\psi}_{2}(-p) \tilde{\psi}_{2}(p)>+N \sin \frac{2 \pi p}{N}<\tilde{\varphi}(-p) \tilde{\varphi}(p)>=0 \\
i N \sin \frac{2 \pi p}{N}<\tilde{\psi}_{1}(-p) \tilde{\psi}_{1}(p)>-<\tilde{D}(-p) \tilde{D}(p)>=0 \\
i<\tilde{\psi}_{2}(-p) \tilde{\psi}_{1}(p)>-\omega^{-p}<\tilde{\varphi}(-p) \tilde{D}(p)>=0 .
\end{array}
$$

Of course these identities can be checked directly using the explicit form of the correlators, but they follow from the exact supersymmetry, endowed with the modified Leibniz rule, discussed in the previous sections. 
The next issue to be investigated will be if exact supersymmetric modified Ward identities hold if the interaction is switched on and loop diagrams come into the game. The standard method based on performing the substitution (6.15) on the functional integral in presence of sources would only lead on the lattice to identities that contain, like (6.18), the explicit variation of the action under such substitution. In fact the action is supersymmetric invariant only if the modified Leibniz rule is applied whenever the variation of a product is taken, and the substitution (6.15) does not account for that. The variation of the action under (6.15) is of order $\frac{1}{N} \equiv a$ and is expected to vanish in the continuum limit, but the question is if this contribution can be exactly accounted for by modifying the Ward identities as shown above for the free theory. This is a difficult task that will be left to future investigation.

\section{Conclusions}

In this paper we have shown in the simple one dimensional example of $N=2$ supersymmetric quantum mechanics, that supersymmetry transformations on the lattice can be defined without any ambiguity with the aid of the modified Leibniz rule if the superfield formalism is consistently used. This clarifies the point raised in [23] and overcomes their objections. The other problem we approached in the paper is the derivation of Ward identities, namely the problem of whether exact supersymmetry is preserved at the quantum level. This is a non trivial problem, because due to the modified Leibniz rule supersymmetry transformations cannot be expressed as a change of variables in the functional integral and the usual derivation of the Ward identities would lead to extra terms ${ }^{6}$, proportional to the lattice spacing. We began to tackle this problem by showing that at least in the case of the theory without interaction (but including the mass term) exact "modified" Ward identities hold, that reflect the modified Leibniz rule of the original symmetry. This is of course a very preliminary step, as we have not been able yet to apply the same procedure to the more interesting case of the theory with interaction. So the problem is still open and left to future investigation. We expect that the situation of higher dimensional models will be similar. We already showed in [7] that the $N=2$ supersymmetric Yang-Mills theory in two dimensions can be formulated on the lattice in a way that the action is exact with respect to the four nilpotent supersymmetry charges, thus ensuring exact supersymmetry under all of them. We also showed that $N=4$ supersymmetric Yang-Mills theory in three dimensions can be formulated on the lattice in a similar way [8]. However in all these cases the exact symmetry is realized classically by using supersymmetry transformations involving a modified Leibniz rule, and a deformation of the Ward identities (if it exist!) will be needed for an exact symmetry at the quantum level ${ }^{7}$.

\section{Acknowledgments}

We would like to thank I. Kanamori and K. Nagata for useful discussions and comments. This work is supported in part by Japanese Ministry of Education, Science, Sports and Culture under the grant number 50169778 and also by Istituto Nazionale di Fisica Nucleare (INFN) research funds.

\footnotetext{
${ }^{6}$ This problem arises whenever modified Leibniz rule appear, as for instance in field theories on noncommutative space-time [28, 29].

${ }^{7}$ Just before completing this paper we received a paper by K. Nagata [30] where this problem is considered in the context of a twisted two dimensional Wess Zumino model.
} 


\section{References}

[1] A. Feo, Nucl. Phys. Proc. Suppl. 119, 198 (2003) [arXiv:hep-lat/0210015].

[2] D. B. Kaplan, E. Katz and M. Unsal, JHEP 0305, 037 (2003) [arXiv:hep-lat/0206019];

A. G. Cohen, D. B. Kaplan, E. Katz and M. Unsal, JHEP 0308, 024 (2003) [arXiv:heplat/0302017] ;

A. G. Cohen, D. B. Kaplan, E. Katz and M. Unsal, JHEP 0312, 031 (2003) [arXiv:heplat/0307012].

[3] S. Catterall and S. Karamov, Phys. Rev. D 65, 094501 (2002) [arXiv:hep-lat/0108024];

S. Catterall and S. Ghadab, JHEP 0405, 044 (2004) [arXiv:hep-lat/0311042] ; ibid. 0610, 063 (2006) [arXiv:hep-lat/0607010] ;

S. Catterall, JHEP 0305, 038 (2003) [arXiv:hep-lat/0301028]; ibid. 0411, 006 (2004) [arXiv:hep-lat/0410052]; ibid. 0506, 027 (2005) [arXiv:hep-lat/0503036]; ibid. 0603, 032 (2006) [arXiv:hep-lat/0602004].

[4] F. Sugino, JHEP 0401, 015 (2004) [arXiv:hep-lat/0311021]; ibid. 0403, 067 (2004) [arXiv:hep-lat/0401017]; ibid. 0501, 016 (2005) [arXiv:hep-lat/0410035];

F. Sugino, Phys. Lett. B 635, 218 (2006) [arXiv:hep-lat/0601024].

[5] A. D'Adda, I. Kanamori, N. Kawamoto and K. Nagata, Nucl. Phys. B 707, 100 (2005) [arXiv:hep-lat/0406029].

[6] A. D'Adda, I. Kanamori, N. Kawamoto and K. Nagata, Nucl. Phys. Proc. Suppl. 140, 754 (2005) [arXiv:hep-lat/0409092].

[7] A. D'Adda, I. Kanamori, N. Kawamoto and K. Nagata, Phys. Lett. B 633, 645 (2006) [arXiv:hep-lat/0507029].

[8] A. D'Adda, I. Kanamori, N. Kawamoto and K. Nagata, Nucl. Phys. B 798, 168 (2008) [arXiv:0707.3533 [hep-lat]];

A. D'Adda, I. Kanamori, N. Kawamoto and K. Nagata, PoS LAT, 271 (2007) [arXiv:0709.0722 [hep-lat]].

[9] P. H. Ginsparg and K. G. Wilson, Phys. Rev. D 25, 2649 (1982).

[10] M. Bonini and A. Feo, JHEP 0409, 011 (2004) [arXiv:hep-lat/0402034] ;

M. Bonini and A. Feo, Phys. Rev. D 71, 114512 (2005) [arXiv:hep-lat/0504010].

[11] P. H. Dondi and H. Nicolai, Nuovo Cim. A 41, 1 (1977).

[12] S. Elitzur, E. Rabinovici and A. Schwimmer, Phys. Lett. B 119, 165 (1982);

T. Banks and P. Windey, Nucl. Phys. B 198 (1982) 226;

S. Cecotti and L. Girardello, Nucl. Phys. B 226, 417 (1983);

N. Sakai and M. Sakamoto, Nucl. Phys. B 229, 173 (1983);

S. Elitzur and A. Schwimmer, Nucl. Phys. B 226, 109 (1983);

I. Ichinose, Phys. Lett. B 122, 68 (1983);

J. Bartels and J. B. Bronzan, Phys. Rev. D 28, 818 (1983);

J. Bartels and G. Kramer, Z. Phys. C 20, 159 (1983);

D. B. Kaplan, Phys. Lett. B 136, 162 (1984); 
R. Nakayama and Y. Okada, Phys. Lett. B 134, 241 (1984);

S. Nojiri, Prog. Theor. Phys. 74, 1124 (1985);

G. Curci and G. Veneziano, Nucl. Phys. B 292, 555 (1987);

M. F. L. Golterman and D. N. Petcher, Nucl. Phys. B 319, 307 (1989).

[13] J. Nishimura, Phys. Lett. B 406, 215 (1997) [arXiv:hep-lat/9701013];

N. Maru and J. Nishimura, Int. J. Mod. Phys. A 13, 2841 (1998) [arXiv:hep-th/9705152];

H. Neuberger, Phys. Rev. D 57, 5417 (1998) [arXiv:hep-lat/9710089];

D. B. Kaplan and M. Schmaltz, Chin. J. Phys. 38, 543 (2000) [arXiv:hep-lat/0002030];

G. T. Fleming, J. B. Kogut and P. M. Vranas, Phys. Rev. D 64, 034510 (2001) [arXiv:heplat/0008009];

M. Harada and S. Pinsky, Phys. Rev. D 71, 065013 (2005) [arXiv:hep-lat/0411024].

[14] I. Montvay, Int. J. Mod. Phys. A 17, 2377 (2002) [arXiv:hep-lat/0112007] ;

R. Kirchner, I. Montvay, J. Westphalen, S. Luckmann and K. Spanderen [DESY-Munster Collaboration], Phys. Lett. B 446, 209 (1999) [arXiv:hep-lat/9810062] ;

I. Campos et al. [DESY-Munster Collaboration], Eur. Phys. J. C 11, 507 (1999) [arXiv:heplat/9903014];

A. Feo, R. Kirchner, S. Luckmann, I. Montvay and G. Munster [DESY-Munster Collaboration], Nucl. Phys. Proc. Suppl. 83, 661 (2000) [arXiv:hep-lat/9909070] ;

F. Farchioni et al. [DESY-Munster-Roma Collaboration], Eur. Phys. J. C 23, 719 (2002) [arXiv:hep-lat/0111008] ;

I. Montvay, Nucl. Phys. Proc. Suppl. 63, 108 (1998) [arXiv:hep-lat/9709080] ;

A. Feo, Phys. Rev. D 70, 054504 (2004) [arXiv:hep-lat/0305020] ;

Y. Taniguchi, Chin. J. Phys. 38, 655 (2000) [arXiv:hep-lat/9906026] ;

K. Itoh, M. Kato, H. Sawanaka, H. So and N. Ukita, JHEP 0302, 033 (2003) [arXiv:heplat/0210049];

A. Feo, P. Merlatti and F. Sannino, Phys. Rev. D 70, 096004 (2004) [arXiv:hep-th/0408214].

[15] T. Onogi and T. Takimi, Phys. Rev. D 72, 074504 (2005) [arXiv:hep-lat/0506014];

H. Suzuki and Y. Taniguchi, JHEP 0510, 082 (2005) [arXiv:hep-lat/0507019];

H. Fukaya, I. Kanamori, H. Suzuki, M. Hayakawa and T. Takimi, Prog. Theor. Phys. 116 (2007) 1117 [arXiv:hep-th/0609049]; H. Fukaya, I. Kanamori, H. Suzuki and T. Takimi, PoS LAT2007 (2007) 264 [arXiv:0709.4076 [hep-lat]];

K. Ohta and T. Takimi, Prog. Theor. Phys. 117, 317 (2007) [arXiv:hep-lat/0611011] ;

T. Takimi, JHEP 0707, 010 (2007) [arXiv:0705.3831 [hep-lat]] ;

H. Suzuki, JHEP 0709, 052 (2007) [arXiv:0706.1392 [hep-lat]];

I. Kanamori, H. Suzuki and F. Sugino, arXiv:0711.2099 [hep-lat]; arXiv:0711.2132 [hep-lat].

[16] W. Bietenholz, Mod. Phys. Lett. A 14, 51 (1999) [arXiv:hep-lat/9807010] ;

K. Fujikawa and M. Ishibashi, Nucl. Phys. B 622, 115 (2002) [arXiv:hep-th/0109156] ;

K. Fujikawa and M. Ishibashi, Phys. Lett. B 528, 295 (2002) [arXiv:hep-lat/0112050] ;

Y. Kikukawa and Y. Nakayama, Phys. Rev. D 66, 094508 (2002) [arXiv:hep-lat/0207013] ;

K. Fujikawa, Phys. Rev. D 66, 074510 (2002) [arXiv:hep-lat/0208015] ;

K. Fujikawa, Nucl. Phys. B 636, 80 (2002) [arXiv:hep-th/0205095] ;

M. Beccaria, M. Campostrini and A. Feo, Phys. Rev. D 69, 095010 (2004) [arXiv:heplat/0402007] ;

M. Beccaria, G. F. De Angelis, M. Campostrini and A. Feo, Phys. Rev. D 70, 035011 (2004) [arXiv:hep-lat/0405016]; 
G. Bergner, T. Kaestner, S. Uhlmann and A. Wipf, Annals Phys. 323, 946 (2008) [arXiv:0705.2212 [hep-lat]].

[17] D. B. Kaplan, Nucl. Phys. Proc. Suppl. 129, 109 (2004) [arXiv:hep-lat/0309099].

[18] S. Catterall, PoS LAT2005, 006 (2006) [arXiv:hep-lat/0509136].

[19] J. Giedt, PoS LAT2006, 008 (2006) [arXiv:hep-lat/0701006].

[20] A. Feo, Mod. Phys. Lett. A 19, 2387 (2004) [arXiv:hep-lat/0410012].

[21] N. Kawamoto and T. Tsukioka, Phys. Rev. D 61 (2000) 105009 [arXiv:hep-th/9905222];

J. Kato, N. Kawamoto and Y. Uchida, Int. J. Mod. Phys. A 19 (2004) 2149 [arXiv:hepth/0310242];

J. Kato, N. Kawamoto and A. Miyake, Nucl. Phys. B 721 (2005) 229 [arXiv:hepth/0502119];

J. Kato and A. Miyake, Mod. Phys. Lett. A 21 (2006) 2569 [arXiv:hep-th/0512269].

[22] K. Nagata, JHEP 0801, 041 (2008) [arXiv:0710.5689 [hep-th]];

K. Nagata and Y. S. Wu, arXiv:0803.4339 [hep-lat].

[23] F. Bruckmann and M. de Kok, Phys. Rev. D 73, 074511 (2006) [arXiv:hep-lat/0603003].

[24] F. Bruckmann, S. Catterall and M. de Kok, Phys. Rev. D 75, 045016 (2007) [arXiv:heplat/0611001].

[25] F. Cooper and B. Freedman, Annals Phys. 146, 262 (1983).

[26] P. H. Damgaard and S. Matsuura, JHEP 0707, 051 (2007) [arXiv:0704.2696 [hep-lat]]; ibid. 0708 (2007) 087 [arXiv:0706.3007 [hep-lat]]; ibid. 0709 (2007) 097 [arXiv:0708.4129 [hep-lat]]; Phys. Lett. B 661 (2008) 52 [arXiv:0801.2936 [hep-th]].

[27] S. Arianos, A. D'Adda, N. Kawamoto and J. Saito, PoS LATTICE2007 (2006) 259 [arXiv:0710.0487 [hep-lat]].

[28] M. Dimitrijevic, V. Radovanovic and J. Wess, JHEP 0712 (2007) 059 [arXiv:0710.1746 [hep-th]].

[29] P. Aschieri, L. Castellani and M. Dimitrijevic, arXiv:0803.4325 [hep-th].

[30] K. Nagata, arXiv:0805.4235 [hep-lat]. 